\title{
VERONESE CURVES AND WEBS: INTERPOLATION
}

\author{
T. B. BOUETOU AND J. P. DUFOUR
}

Received 11 June 2006; Revised 30 September 2006; Accepted 19 October 2006

We review basic results, essentially due to J. Turiel, concerning the link between classical multidimensional webs and Veronese webs.

Copyright (c) 2006 Hindawi Publishing Corporation. All rights reserved.

\section{Introduction}

In this survey, we will be interested in Veronese webs (particular case of one parameter families of foliations), as defined by $[8,16]$, and ordinary webs (finite families of foliations in general position), as defined by Blaschke, Akivis and Goldberg [1-5,9]. If we look at the literature about webs, these two domains were developed apparently independently. Our main goal is to establish the link between the two domains.

\subsection{Classical webs.}

Definition 1.1. A k-web of codimension $c$ over a manifold $V$ is a family of $k$ foliations $\mathfrak{F}_{1}, \mathfrak{F}_{2}, \ldots, \mathfrak{F}_{k}$ of $V$, all of codimension $c$, in "general position." This last condition means that if $\mathfrak{F}_{i}(m)$ denotes the tangent plane to $\mathfrak{F}_{i}$ at the point $m$ (the contact element to $\mathfrak{F}_{i}$ at $m$ ), then the $c$-codimensional subspaces $\mathfrak{F}_{1}(m), \mathfrak{F}_{2}(m), \ldots, \mathfrak{F}_{k}(m)$ of $T_{m} V$ are in general position (as transverse as possible).

Classical examples are k-webs of $\mathbb{R}^{2}$, that is, systems of $k$ families of curves in a plane.

We will simply call 3 -web any 3 -web of codimension $c$ on a $2 c$-dimensional manifold. General position means here that we have $\mathfrak{F}_{i}(m) \cap \mathfrak{F}_{j}(m)=\{0\}$ for $i \neq j$ and at any $m$. These webs are related to binary laws: if $(x, y) \mapsto x \circ y$ is a smooth binary law on the manifold $M$, then we can associate the three foliations $x=C^{t e}, y=C^{t e}$, and $x \circ y=C^{t e}$ on $M \times M$; with further hypotheses, this gives a 3-web (e.g., if $x \circ y$ is a Lie group law...). The 3 -webs were intensively investigated in $[1-5,7,10,11]$. 
2 Veronese curves and webs: interpolation

More generally, a $(p+1)$-web will be a $(p+1)$-web of codimension $c$ on a manifold of dimension $p c$. Here general position means that, for all $m \in V$, we have

$$
\mathfrak{F}_{1}(m) \cap \cdots \cap \mathfrak{F}_{i-1}(m) \cap \mathfrak{F}_{i+1}(m) \cap \cdots \cap \mathfrak{F}_{p+1}(m)=\{0\},
$$

for any $i=1, \ldots, p+1$.

Remark 1.2. A $p$-web of codimension $c$ on a manifold of dimension $p c$ is locally trivial, that is, we can find local coordinates

$$
x_{1}^{1}, \ldots, x_{c}^{1}, \ldots, x_{1}^{p}, \ldots, x_{c}^{p}
$$

where $\mathfrak{F}_{i}$ is given by the equations $\left\{x_{1}^{i}=C^{t e}, \ldots, x_{c}^{i}=C^{t e}\right\}$. So the first webs which have an interesting local geometry are the above-defined $(p+1)$-webs.

\subsection{Veronese webs.}

Definition 1.3. Let $V$ be a real vector space of dimension $(n+1)$. A Veronese curve in the projective space $\mathbb{P}(V)$ is a map

$$
\gamma: \mathbb{P}^{1} \mathbb{R} \longrightarrow \mathbb{P} V
$$

which is the quotient of a map of the type

$$
(x, y) \longmapsto x^{n} v_{n}+x^{n-1} y v_{n-1}+\cdots+y^{n} v_{0}
$$

where $\left(v_{0}, v_{1}, \ldots, v_{n}\right)$ is a base in $V$.

Definition 1.4 (see $[8,13,18]$ ). A Veronese web of codimension $c$ on a manifold $V$ of dimension $p c$ is a one-parameter family of foliations $\left(\mathfrak{F}_{t}\right)_{t \in \mathbb{P}^{1} \mathbb{R}}$ of codimension $c$ on $V$ such that, for all $m$ in $V$, the contact element $\mathfrak{F}_{t}(m)$ is given by

$$
\alpha_{t}^{1}=0, \ldots, \alpha_{t}^{c}=0
$$

with

$$
\alpha_{t}^{i}=\gamma_{0}^{i}+t \gamma_{1}^{i}+t^{2} \gamma_{2}^{i}+\cdots+t^{p-1} \gamma_{p-1}^{i},
$$

where $\left(\gamma_{j}^{i}\right)_{\substack{i=1, \ldots, c \\ j=0, \ldots, p-1}}$ form a local coframe; that is,

$$
\gamma_{0}^{1}, \ldots, \gamma_{p-1}^{1}, \gamma_{0}^{2}, \ldots, \gamma_{p-1}^{2}, \ldots, \gamma_{0}^{c}, \ldots, \gamma_{p-1}^{c}
$$

are differential forms, defined in a neighborhood of $m$ such that

$$
\gamma_{0}^{1}(m), \ldots, \gamma_{p-1}^{1}(m), \ldots, \gamma_{0}^{c}(m), \ldots, \gamma_{p-1}^{c}(m)
$$

is a basis of $T_{m}^{\star} V$. 
Gelfand and Zakharevich [8] defined Veronese webs of codim $1(c=1)$ and the notion was generalized by A. Panasyuk and J. Turiel. In the sequel, we will sketch the way these notions appeared.

In the bihamiltonian "mechanics," we study pencil of Poisson structures $\Pi_{0}+t \Pi_{\infty}$ over a manifold $W$. This means that (see [8]) $\Pi_{t}=\Pi_{0}+t \Pi_{\infty}$ are Poisson structures for all $t$ and $\Pi_{\infty}$ is also a Poisson structure. It is equivalent to say that $\Pi_{0}$ and $\Pi_{\infty}$ are Poisson structures with $\left[\Pi_{0}, \Pi_{\infty}\right]=0([\cdot, \cdot]$ is the so-called Schouten bracket $)$; in that case we say that $\Pi_{0}$ and $\Pi_{\infty}$ are "compatible."

For some time it was believed that any integrable Hamiltonian system was a bihamiltonian system, that is, there exists a second Poisson structure compatible with the Poisson structure related to the initial symplectic structure, which should be invariant by the Hamiltonian field. The correct idea is that any bihamiltonian system is integrable but Brouzet [6] has shown that the former belief was wrong. Nevertheless the classical integrable systems are all bihamiltonian.

Turiel [15] has classified the pairs of compatible Poisson structures $\left(\Pi_{0}, \Pi_{\infty}\right)$ with $\Pi_{0}$ symplectic (here we are in an even-dimensional situation). On the other hand, I. Gelfand and I. Zakharevich were the first to investigate the odd-dimensional case. Precisely, they consider a pencil

$$
\Pi_{t}=\Pi_{0}+t \Pi_{\infty}
$$

on a $2 p-1$-dimensional manifold such that $\Pi_{t}$ is, for all $t$, of maximum rank $(2 p-2)$. The symplectic foliation $\mathfrak{F}_{t}$ of $\Pi_{t}$ is then of codimension 1 and locally given by the zeros of a form $\alpha_{t}$. It is not yet a Veronese foliation in the sense of Definition 1.4, but we will explain hereafter that it is the case up to a quotient.

In fact, we have the following local models:

$$
\begin{aligned}
& \Pi_{0}(m)=e_{1} \wedge f_{1}+e_{2} \wedge f_{2}+\cdots+e_{p-1} \wedge f_{p-1} \\
& \Pi_{\infty}(m)=f_{1} \wedge e_{2}+f_{2} \wedge e_{3}+\cdots+f_{p-1} \wedge e_{p}
\end{aligned}
$$

where $e_{1}, \ldots, e_{p}, f_{1}, \ldots, f_{p-1}$ is a well-chosen base of $T_{m} V$; denote by $e_{1}^{*}, \ldots, e_{p}^{*}, f_{1}^{*}, \ldots, f_{p-1}^{*}$ the dual base of $T_{m}^{*} V$. The distribution $\mathfrak{F}_{t}(m)$ is the symplectic foliation of

$$
\Pi_{t}(m)=e_{1} \wedge f_{1}+e_{2} \wedge f_{2}+\cdots+e_{p-1} \wedge f_{p-1}+t\left(f_{1} \wedge e_{2}+f_{2} \wedge e_{3}+\cdots+f_{p-1} \wedge e_{p}\right) .
$$

It is easy to see that the distribution annihilates the form

$$
\beta_{t}=e_{p}^{*}+t e_{p-1}^{*}+\cdots+t^{p-1} e_{1}^{*}
$$

and that $\mathfrak{F}_{t}(m)$ contains $\left\langle f_{1}, \ldots, f_{p-1}\right\rangle$.

Take a submanifold $V$ of dimension $p$ transverse to $\left\langle f_{1}, \ldots, f_{p-1}\right\rangle$, the traces of $\mathfrak{F}_{t}$ on $V$ form a Veronese web of codim 1 defined by

$$
\alpha_{t}=e_{p}^{*}+t e_{p-1}^{*}+\cdots+t^{p-1} e_{1}^{*} .
$$

The theory initiated by Gelfand-Zakharevitch and ended by J. Turiel says that the local invariants of the pair $\left(\Pi_{0}, \Pi_{\infty}\right)$ are the local invariants of this Veronese foliation restricted 
4 Veronese curves and webs: interpolation

to $V$. Latter, the pairs $\left(\Pi_{0}, \Pi_{\infty}\right)$ such that $\Pi_{t}$ is of constant corank $c>1$ where investigated and, by the use of the same method, one obtain Veronese webs in the sense of Definition 1.4.

\section{Link between $(p+1)$-webs and Veronese webs}

Let $\left(\mathfrak{F}_{t}\right)_{t}$ be a Veronese web of codimension $c$ over the $p c$-dimensional manifold $V$. Assume that $t_{1}, \ldots, t_{p+1}$ are two by two distinct then $\left(\mathfrak{F}_{t_{i}}\right)_{i=1, \ldots, p+1}$ gives a $(p+1)$-web. In fact, $\left(\mathfrak{F}_{t_{i}}\right)$ is locally given by

$$
\alpha_{t_{i}}^{1}=0, \ldots, \alpha_{t_{i}}^{c}=0
$$

with

$$
\alpha_{t}^{i}=\gamma_{0}^{i}+\gamma_{1}^{i} t+\cdots+\gamma_{p-1}^{i} t^{p-1}
$$

Since

$$
\left|\begin{array}{cccc}
1 & t_{i_{1}} & \cdots & t_{i_{1}}^{p-1} \\
1 & t_{i_{2}} & \cdots & t_{i_{2}}^{p-1} \\
\vdots & \vdots & \vdots & \vdots \\
1 & t_{i_{p}} & \cdots & t_{i_{p}}^{p-1}
\end{array}\right|
$$

is a Van Der Monde determinant, it is clear that

$$
\alpha_{t_{i_{1}}}^{1}, \ldots, \alpha_{t_{i_{1}}}^{c}, \ldots, \alpha_{t_{i_{p}}}^{1}, \ldots, \alpha_{t_{i_{p}}}^{c}
$$

form a base of $T^{*} V$ for all $i_{1}, \ldots, i_{p}$, two by two distinct with $\left\{i_{1}, \ldots, i_{p}\right\} \subset\{1, \ldots, p+1\}$, therefore we have the condition of general position.

The most difficult problem is the passage from the $(p+1)$-webs to Veronese webs. We have a problem of interpolation of $\left(\mathfrak{F}_{i}\right)_{i=1, \ldots, p+1}$ to a curve $\left(\mathfrak{F}_{t}\right)_{t \in \mathbb{P} 1 \mathbb{R}}$ having good properties. We decompose this into two problems.

Algebraic interpolation. Given $p+1$ subspaces of codimension $c$ in a $p c$-dimensional vector space $V$ in general position, find a natural curve of subspaces of codimension $c$ in $V$ passing through the given $p+1$ subspaces. It is a problem in $G_{c}(V)$ the Grassmannian of subspaces of codimension $c$ in $V$. Let us assume that this problem has a unique solution. Given $p+1$ distributions of codimension $c$ on a manifold of dimension $p c$, there would exist a natural method to interpolate these $p+1$ distributions $\mathfrak{F}_{1}, \mathfrak{F}_{2}, \ldots, \mathfrak{F}_{p+1}$ in a curve $\mathfrak{F}_{t}$ of distributions.

Integrability. Under which condition these distributions are integrable? For example, is the integrability of $\mathfrak{F}_{1}, \mathfrak{F}_{2}, \ldots, \mathfrak{F}_{p+1}$ sufficient to guaranty that of $\mathfrak{F}_{t}$ for all $t$ ?

In the next sections, we will investigate these questions. 


\section{Interpolation of a finite family of subspaces}

Let $V$ be a vector space and $G_{c}(V)$ the Grassmannian of its codimension $c$ subspaces. We put $N=\operatorname{dim} V-c$ and denote by $S_{c}(V)$ the open subset of $V^{N}$ formed by $N$-uples of linearly independent vectors of $V$. Let $\beta: \mathbb{P}^{1} \mathbb{R} \rightarrow G_{c}(V)$; it is said to be a degree $q$ curve if it pulls back as follows:

$$
\hat{\beta}: \mathbb{R}^{2} \backslash 0 \longrightarrow S_{c}(V), \quad \hat{\beta}(x, y)=\left(\beta_{1}(x, y), \ldots, \beta_{N}(x, y)\right),
$$

where $\beta_{i}$ has the form

$$
\beta_{i}=\sum_{j} \beta_{i}^{j}(x, y) e_{j}
$$

$\left(e_{j}\right)_{j}$ is a basis of $V$, and $\beta_{i}^{j}$ are homogeneous polynomials of degree $q$; we have the following commutative diagram:

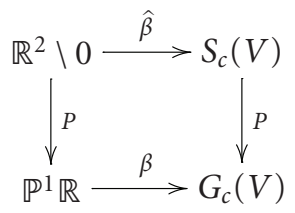

where $P$ are canonical projections $\left(P\left(v_{1}, \ldots, v_{n}\right)=\left\langle v_{1}, \ldots, v_{n}\right\rangle\right)$.

Let $F_{1}, \ldots, F_{p+1}$ be given points of $G_{c}(V)$; we will say that $\beta: \mathbb{P}^{1} \mathbb{R} \rightarrow G_{c}(V)$ is a minimal interpolation of $\left(F_{1}, \ldots, F_{p+1}\right)$ if $\beta$ is a curve of minimal degree $q$ passing through $F_{1}, \ldots, F_{p+1}$.

It is a difficult problem to find such minimal interpolations and see if they are unique: in general it is wrong. Furthermore, these curves are not independent of the choice of the parametrization: the sequences of $t_{i}$ such that $\beta\left(t_{i}\right)=F_{i}$. In the sequel, we will show that there are unique minimal interpolations in two important cases:

(i) the case where $\operatorname{dim} V=p c\left(\operatorname{dim} F_{i}=(p-1) c\right)$,

(ii) the case where $\operatorname{dim} V=p N\left(\operatorname{dim} F_{i}=N\right)$.

In the first case, the minimal interpolations are pencils, that is, degree 1 curves, of $c$ codimensional subspaces; in the second case we recover Veronese curves and their generalization. Moreover, these cases are dual to each other.

3.1. Interpolation by pencils. In this section, we deal with a family $F_{1}, \ldots, F_{p+1}$ of $c$ codimensional subspaces of the $p c$-dimensional vector space $V$. We suppose that this family is in general position: this means that, for every $i$, we have $F_{1} \cap \cdots \cap F_{i-1} \cap F_{i+1} \cap$ $\cdots \cap F_{p+1}=\{0\}$.

Fix a system of linear coordinates $\left(x_{1}^{1}, \ldots, x_{1}^{c} ; x_{2}^{1}, \ldots, x_{2}^{c} ; \ldots ; x_{p}^{1}, \ldots, x_{p}^{c}\right)$ such that the equations of $F_{i}$ are $x_{i}^{1}=0, \ldots, x_{i}^{c}=0$ for $i=1, \ldots, p$, and $F_{p+1}$ has equations $\sum_{i} x_{i}^{1}=0, \ldots$, $\sum_{i} x_{i}^{c}=0$. We denote by

$$
\left(e_{1}^{1}, \ldots, e_{1}^{c} ; e_{2}^{1}, \ldots, e_{2}^{c} ; \ldots ; e_{p}^{1}, \ldots, e_{p}^{c}\right)
$$

the corresponding basis. 
We fix also a system $t_{1}, \ldots, t_{p}$ of two by two different real numbers.

A pencil of $c$-dimensional subspaces is a degree 1 curve $\beta$ of $c$-dimensional subspaces of $V$ : this means that $\beta$ pulls back as a curve $\hat{\beta}: \mathbb{R}^{2} \backslash 0 \rightarrow S_{c}(V)$ with $\hat{\beta}(x, y)=\left(x a^{1}+\right.$ $\left.y b^{1}, \ldots, x a^{(p-1) c}+y b^{(p-1) c}\right)$, where $a^{j}$ and $b^{j}$ are vectors of $V$. With the identification $t \equiv$ $[t: 1]$, we can write $\beta(t)=(G-t \mathrm{Id}) \beta(\infty)$, where $\beta(\infty)$ is the space generated by the $a^{j}$ and $G: V \rightarrow V$ is any linear map such that $G\left(a^{j}\right)=-b^{j}$ for every $j$.

We want to interpolate the $F_{i}$ by such a pencil. More precisely, we want a pencil $\beta$ with $\beta\left(t_{i}\right)=F_{i}$, for $i=1, \ldots, p$ and $\beta(\infty)=F_{p+1}$. A simple solution is obtained by choosing $G$ such that $G\left(e_{i}^{j}\right)=t_{i} e_{i}^{j}$ for every $i=1, \ldots, p$ and $j=1, \ldots, c$ we have

$$
\beta(\infty)=\left\langle e_{p}^{j}-e_{k}^{j} ; k=1, \ldots, p-1 ; j=1, \ldots, c\right\rangle,
$$

then

$$
\beta(t)=\left\langle\left(t_{p}-t\right) e_{p}^{j}-\left(t_{k}-t\right) e_{k}^{j} ; k=1, \ldots, p-1 ; j=1, \ldots, c\right\rangle
$$

and it is easy to see that $\beta\left(t_{i}\right)$ has equations $x_{i}^{1}=0, \ldots, x_{i}^{c}=0$.

In the sequel, we will investigate the uniqueness of this pencil.

First we will suppose there is another linear map $G^{\prime}$ with $\left(G^{\prime}-t_{i} \mathrm{Id}\right)\left(F_{p+1}\right)=F_{i}$ for every $i=1, \ldots, p$. Put

$$
G^{\prime}\left(e_{p}^{j}-e_{k}^{j}\right):=u_{k}^{j}=\sum_{r=1 \cdots c, s=1 \cdots p} a_{k s}^{j r} e_{s}^{r}
$$

Then equations $x_{r}^{j}\left(u_{i}^{s}-t_{r}\left(e_{p}^{s}-e_{i}^{s}\right)\right)=0$ for every $s, j=1, \ldots, c, i=1, \ldots, p-1$ and $r=$ $1, \ldots, p$ lead to

$$
u_{k}^{j}=t_{p} e_{p}^{j}-t_{k} e_{k}^{j}
$$

for every $j=1, \ldots, c$ and $k=1, \ldots, p-1$. So the pencil attached to $G^{\prime}$ is exactly $\beta$ (the one attached to $G)$.

We can remark that the difference $\Delta=G^{\prime}-G$ is a linear mapping of $V$ such that $\Delta\left(e_{p}^{j}-\right.$ $\left.e_{k}^{j}\right)=0$ for every $j$ and $k$. So $\Delta$ is characterized by the fact that there are arbitrary vectors $v^{1}, \ldots, v^{c}$ of $V$ with $\Delta\left(e_{k}^{j}\right)=v^{j}$, for every $j$ and $k$. In particular, we can always manage such that $G^{\prime}$ is invertible: if the $t_{i}$ are all nonzero, then $G$ is invertible; if, for example, $t_{1}$ vanishes, we can choose $v^{j}=e_{1}^{j}$ for every $j$.

Next we remark that coordinates $\left(x_{i}^{j}\right)_{i=1, \ldots, p}^{j=1, \ldots, c}$ are unique up to a linear change of the form $\left(x_{i}^{j}\right)^{\prime}=\sum_{s=1, \ldots, c} a_{s}^{j} x_{i}^{s}$; this means that the matrix of this linear change is a $p c \times p c$ matrix which has only null terms except $p$ diagonal $c \times c$ blocs all equal to $A=\left(a_{s}^{r}\right)_{r, s=1, \ldots, c}$. This induces that $\beta$ does not depend on the particular choice of the adapted coordinates $x_{i}^{j}$.

Finally, we want to see how this interpolation depends on the parametrization, that is, on the sequence $t_{1}, \ldots, t_{p}$. First of all, remark that, if $\beta$ is a pencil as above, then we can perform a projective transform on the parameter space $\mathbb{P}^{1} \mathbb{R}$ and we keep the pencil. This allows us to impose the values at three different points: this justifies a posteriori 
the particular choice of $\beta(\infty)$ in the preceding calculations. We could also have fixed two other values, for example, $t_{1}=0$ and $t_{2}=1$ (imposing $\beta(0)=F_{1}$ and $\beta(1)=F_{2}$ ). The following lemma says that two pencils which interpolate $F_{1}, \ldots, F_{p+1}$ are the same if and only if the sequences of parameters $\tau_{1}, \ldots, \tau_{p+1}$, where these pencils pass respectively at $F_{1}, \ldots, F_{p+1}$, are the same up to a projective transformation of $\mathbb{P}^{1} \mathbb{R}$.

Lemma 3.1. Let $\beta$ and $\beta^{\prime}$ be two pencils, interpolating $F_{1}, \ldots, F_{p+1}$, such that

$$
\beta(\infty)=\beta^{\prime}(\infty)=F_{p+1}, \quad \beta(0)=\beta^{\prime}(0)=F_{1}, \quad \beta(1)=\beta^{\prime}(1)=F_{2} .
$$

Let $t_{i}$ and $t_{i}^{\prime}$ for $i=3, \ldots, p$ be the values of the parameters such that $F_{i}=\beta\left(t_{i}\right)=\beta^{\prime}\left(t_{i}^{\prime}\right)$. Then $\beta$ and $\beta^{\prime}$ have the same image $\left(\left\{\beta(t) ; t \in \mathbb{P}^{1} \mathbb{R}\right\}=\left\{\beta^{\prime}(t) ; t \in \mathbb{P}^{1} \mathbb{R}\right\}\right)$ if and only if $t_{i}=t_{i}^{\prime}$ for every $i=3, \ldots, p$.

Proof. The preceding calculations give the "if" part. To prove the converse, we suppose that, for each $t \in \mathbb{P}^{1} \mathbb{R}$, there is $t^{\prime} \in \mathbb{P}^{1} \mathbb{R}$, with

$$
\beta(t)=\beta^{\prime}\left(t^{\prime}\right) .
$$

Formula (3.6) gives

$$
\begin{aligned}
& \left\langle\left(t_{p}-t\right) e_{p}^{j}-\left(t_{k}-t\right) e_{k}^{j} ; k=1, \ldots, p-1 ; j=1, \ldots, c\right\rangle \\
& \quad=\left\langle\left(t_{p}^{\prime}-t^{\prime}\right) e_{p}^{j}-\left(t_{k}^{\prime}-t^{\prime}\right) e_{k}^{j} ; k=1, \ldots, p-1 ; j=1, \ldots, c\right\rangle,
\end{aligned}
$$

for every $t$. From this, we deduce equations

$$
\left(t_{p}-t\right)\left(t_{k}^{\prime}-t^{\prime}\right)=\left(t_{p}^{\prime}-t^{\prime}\right)\left(t_{k}-t\right)
$$

so relations

$$
t^{\prime}=t \frac{t_{p}^{\prime}-t_{k}^{\prime}}{t_{p}-t_{k}}+\frac{t_{p} t_{k}^{\prime}-t_{p}^{\prime} t_{k}}{t_{p}-t_{k}}
$$

for $k=1, \ldots, p-1$. Then hypothesis $t_{1}=t_{1}^{\prime}$ and $t_{2}=t_{2}^{\prime}$ imply $t_{k}=t_{k}^{\prime}$ for every $k$.

3.2. Veronese interpolations. To each subspace $F$ of the vector space $V$, we associate its annihilator $F^{\circ}$ which is the subset of $V^{*}$ formed by the linear forms on $V$ which vanish on $F$. Now if $\beta$ is an interpolation of the family of subspaces $F_{1}, \ldots, F_{p+1}$ of $V$, then $\beta^{\circ}$, defined by $\beta^{\circ}(t)=(\beta(t))^{\circ}$, is an interpolation of the family of subspaces $F_{1}^{\circ}, \ldots, F_{p+1}^{\circ}$.

Now suppose that $V$ has dimension $p N$ and the $F_{i}$ have dimension $N$. Then $F_{i}^{\circ}$ have codimension $c:=N$ and the preceding section gives pencil interpolations, in the general position cases, for $F_{1}^{\circ}, \ldots, F_{p+1}^{\circ}$. Denote by $\gamma$ such a pencil; we have (formula (3.6))

$$
\gamma(t)=\left\langle\left(t_{p}-t\right) \alpha_{p}^{j}-\left(t_{k}-t\right) \alpha_{k}^{j} ; k=1, \ldots, p-1 ; j=1, \ldots, c\right\rangle,
$$

for a good basis $\left(\alpha_{k}^{j}\right)_{i, k}$ of $V^{*}$ and a parametrization such that

$$
\gamma(\infty)=F_{p+1}^{\circ}, \quad \gamma\left(t_{i}\right)=F_{i}^{\circ}
$$


for $i=1, \ldots, p$. If $\left(e_{k}^{j}\right)_{i, k}$ is the dual basis to $\left(\alpha_{k}^{j}\right)_{i, k}$, we have

$$
\gamma^{\circ}(t)=\left\langle\sum_{i=1}^{p}\left(t_{1}-t\right) \cdots\left(t_{i-1}-t\right)\left(t_{i+1}-t\right) \cdots\left(t_{1}-t\right) e_{i}^{j} ; j=1, \ldots, c\right\rangle .
$$

So we get a degree $p-1$ interpolation of $F_{1}, \ldots, F_{p+1}$. In the case where $N(=c)=1$, we can prove that $\gamma^{\circ}$ gives a Veronese curve in $\mathbb{P}(V)$. For this reason, we call these $\gamma^{\circ}$ Veronese interpolations, even in the case $N>1$. Uniqueness properties of pencil interpolations translate into corresponding uniqueness properties for Veronese interpolations.

Example 3.2. For $p=2$, a Veronese interpolation is also a pencil.

Example 3.3. For $p=3$ and $N=1$, a Veronese interpolation is a degree 2 curve in a projective plane: it is a conic. We recover that there are conics passing by four given points, and the Lemma 3.1 is the generalization of the classical result which says that such a conic is characterized by the cross-ratio of these four points on the conic.

\section{Integrability of distributions}

4.1. Distributions. The results of the preceding section are purely algebraic but they pass to smooth distributions on manifolds. For example, when we have $(p+1)$ smooth distributions $\mathfrak{F}_{1}, \ldots, \mathfrak{F}_{p+1}$ of codimension $c$, in general position, on a manifold $W$ of dimension $p c$, we can work point by point in each tangent space $T_{m} W$ to construct the distribution $\mathfrak{F}_{t}$ which interpolate them. The uniqueness of this procedure ensures their smoothness. To be coherent with the vocabulary of our second section, we call these 1-parameter families of distribution Veronese distributions.

In the neighborhood $U$ of each point $m$, we have a family of operators $G(m)$, depending smoothly on $m$, such that

$$
\mathfrak{F}_{t}(m)=(G(m)-t I) \mathfrak{F}_{\infty}(m) .
$$

4.2. Integrability theorem. In this section, we will give a short proof of the following theorem of Panasyuk (see [14]).

Theorem 4.1. Let $\left(\mathfrak{F}_{t}\right)_{t}$ be a Veronese distribution on a pc-dimensional manifold $W$. The distribution $\mathfrak{F}_{t}$ is integrable for any $t$ if and only if there exist $p+2$ values of $t$ for which $\mathfrak{F}_{t}$ is integrable.

This theorem is not evident in the covariant version, that is, when we define distributions as zeros of set of forms $\alpha^{1}(t), \ldots, \alpha^{c}(t)$ using the Frobenius theorem, the integrability of $\mathfrak{F}_{t}$, for any $t$, is locally equivalent to

$$
d \alpha^{i}(t) \wedge \alpha^{1}(t) \wedge \cdots \wedge \alpha^{c}(t) \equiv 0,
$$

for all $t$. This gives a polynomial equation of degree $(c+1) p$ in $t$. It will vanish identically if it vanishes at $(c+1) p+1$ values of $t$ which is, in general, bigger than $p+2$. 
It is not also evident in contravariant version, that is, when we define distribution by means of vector fields: $\mathfrak{F}_{t}$ is integrable, for any $t$, if and only if, for any $t$,

$$
\left[X_{i}(t), X_{j}(t)\right] \wedge X_{1}(t) \wedge \cdots \wedge X_{(p-1) c}(t)=0,
$$

by denoting $\mathfrak{F}_{t}=\left\langle X_{1}, \ldots, X_{(p-1) c}\right\rangle$ where $X_{1}, \ldots, X_{(p-1) c}$ form a local basis. This gives a polynomial equation of degree $2+(p-1) c$. It will vanish identically if it vanishes at $3+$ $(p-1) c$ values of $t$, still bigger than $p+2$.

Proof. It is sufficient to work locally in a neighborhood of any point of $W$ : we choose invertible operators $G(m)$, depending smoothly on $m$, with $\mathfrak{F}_{t}(m)=(G(m)-t I) \mathfrak{F}_{\infty}(m)$. We choose also a family of vector fields $\left(v_{1}, \ldots, v_{(p-1) c}\right)$ which generates locally $\mathfrak{F}_{\infty}(m)$.

The integrability of $\mathfrak{F}_{t}$ is given by equation

$$
\left[(G-t I) v_{i},(G-t I) v_{j}\right]=\sum_{k} \theta_{i j}^{k}(G-t I) v_{k}
$$

for any $i, j$.

Let us assume that $\left(\mathfrak{F}_{t}\right)$ is integrable for $p+2$ values of $t$; we can assume that it is true for $t=0, \infty$ and $t_{1}, \ldots, t_{p}$ two by two distinct. This implies relations

$$
\left[v_{i}, v_{j}\right]=\sum_{k} \alpha_{i j}^{k} v_{k}
$$

$($ for $t=\infty)$,

$$
\left[G v_{i}, G v_{j}\right]=\sum_{k} \beta_{i j}^{k} G\left(v_{k}\right)
$$

(for $t=0)$. We have

$$
\left[(G-t I) v_{i},(G-t I) v_{j}\right]=\left[G v_{i}, G v_{j}\right]-t \Delta\left(v_{i}, v_{j}\right)+t^{2}\left[v_{i}, v_{j}\right]
$$

with $\Delta\left(v_{i}, v_{j}\right)=\left[G v_{i}, v_{j}\right]+\left[v_{i}, G v_{j}\right]$. We introduce the Nijenhuis torsion $N_{G}[12]$ of $G$ :

$$
N_{G}\left(v_{i}, v_{j}\right)=\left[G v_{i}, G v_{j}\right]-G \Delta\left(v_{i}, v_{j}\right)+G^{2}\left[v_{i}, v_{j}\right] .
$$

Then we get

$$
\Delta\left(v_{i}, v_{j}\right)=G^{-1}\left[G v_{i}, G v_{j}\right]+G\left[v_{i}, v_{j}\right]-G^{-1} N_{G}\left(v_{i}, v_{j}\right)
$$

Thus the first member of formula (4.7) becomes

$$
\begin{aligned}
\left(I-t G^{-1}\right)\left[G v_{i}, G v_{j}\right]-t\left((G-t I)\left[v_{i}, v_{j}\right]\right)+t G^{-1} N_{G}\left(v_{i}, v_{j}\right) \\
\quad=G^{-1}(G-t I)\left[G v_{i}, G v_{j}\right]-t\left((G-t I)\left[v_{i}, v_{j}\right]\right)+t G^{-1} N_{G}\left(v_{i}, v_{j}\right) \\
\quad=G^{-1}(G-t I) \sum_{k} \beta_{i j}^{k} G\left(v_{k}\right)-t\left((G-t I) \sum_{k} \alpha_{i j}^{k} v_{k}\right)+t G^{-1} N_{G}\left(v_{i}, v_{j}\right) \\
=(G-t I) \sum_{k} \gamma_{i j}^{k}(t) v_{k}+t G^{-1} N_{G}\left(v_{i}, v_{j}\right)
\end{aligned}
$$


with $\gamma_{i j}^{k}(t)=\beta_{i j}^{k}-t \alpha_{i j}^{k}$. The integrability for $t=t_{1}, t_{2}, \ldots, t_{p}$ gives us equations

$$
t_{r} G^{-1} N_{G}\left(v_{i}, v_{j}\right)=\left(G-t_{r} I\right)\left(\sum_{k} \mu_{i j}^{k}\left(t_{r}\right) v_{k}\right)
$$

for $r=1, \ldots, p$.

Therefore $G^{-1} N_{G}\left(v_{i}, v_{j}\right)$ is in $\bigcap_{r=1}^{p} \mathfrak{F}_{t_{r}}$. As we have

$$
\bigcap_{r=1}^{p} \mathfrak{F}_{t_{r}}=\{0\},
$$

( $t_{r}$ two by two distinct), we can conclude that

$$
G^{-1} N_{G}\left(v_{i}, v_{j}\right)=0,
$$

then $N_{G}\left(v_{i}, v_{j}\right)=0$, for any $i, j$. So we have, for any $t$,

$$
\left[(G-t I) v_{i},(G-t I) v_{j}\right]=\sum_{k} \theta_{i j}^{k}(G-t I) v_{k}
$$

So we obtain the integrability of each $\mathfrak{F}_{t}$.

Remark 4.2. In his study of Veronese webs (see $[16,17])$, Turiel invented the above technics. He fixes $p+1$ foliations of the family, say $\mathfrak{F}_{\infty}$ and $\mathfrak{F}_{t_{i}}$, for $i=1, \ldots, p$. The $p$ distributions $\mathfrak{H}_{i}$, defined by

$$
\mathfrak{H}_{i}(m)=\bigcap_{j=1, \ldots, i-1, i+1, \ldots, p} \mathfrak{F}_{j}(m),
$$

for $i=1 \ldots, p$, are integrable and decompose, at each point $m$, the tangent space in a direct sum. So the operator $G$ defined by $G=t_{i} I$ in restriction to every $\mathfrak{H}_{i}$, has a null Nijenhuis torsion. This simplifies the above calculations; nevertheless the integrability of $\mathfrak{F}_{\infty}$ and $\mathfrak{F}_{t_{i}}$, for $i=1, \ldots, p$ does not ensure that of the whole family because either $G$ is not invertible or we do not know if $\mathfrak{F}_{0}$ is integrable. As we saw in the paragraph preceding Lemma 3.1, we can replace our $G$ with $G^{\prime}=G+\Delta$ for a well-chosen $\Delta$; doing this we can get $G^{\prime}$ with nonzero Nijenhuis torsion.

\section{References}

[1] M. A. Akivis, Three-webs of multidimensional surfaces, Trudy Geometricheskogo Seminara 2 (1969), 7-31 (Russian).

[2] M. A. Akivis and V. V. Goldberg, Differential geometry of webs, Handbook of Differential Geometry, Vol. I (F. J. E. Dillen and L. C. A. Verstraelen, eds.), chapter 1, North-Holland, Amsterdam, 2000, pp. 1-152.

[3] M. A. Akivis and A. M. Shelekhov, Geometry and Algebra of Multidimensional Three-Webs, Mathematics and Its Applications (Soviet Series), vol. 82, Kluwer Academic, Dordrecht, 1992.

[4] W. Blaschke, Einführung in die geometrie der waben, Birkhäuser, Basel und Stuttgart, 1955.

[5] W. Blaschke and G. Bol, Geometrie der Gewebe, Grundlehren der Mathematischen Wissenschaften, Springer, Berlin, 1938. 
[6] R. Brouzet, About the existence of recursion operators for completely integrable Hamiltonian systems near a Liouville torus, Journal of Mathematical Physics 34 (1993), no. 4, 1309-1313.

[7] J. P. Dufour, Introduction aux tissus, Séminaire Gaston Darboux de Géométrie et Topologie Différentielle, 1990/1991 (Montpellier, 1990/1991), Univ. Montpellier II, Montpellier, 1992, pp. $55-76$.

[8] I. M. Gelfand and I. Zakharevich, Webs, Veronese curves, and bi-Hamiltonian systems, Journal of Functional Analysis 99 (1991), no. 1, 150-178.

[9] V. V. Goldberg, Theory of Multicodimensional $(n+1)$-Webs, Mathematics and Its Applications, vol. 44, Kluwer Academic, Dordrecht, 1988.

[10] J. Grifone and E. Salem (eds.), Web Theory and Related Topics, World Scientific, New Jersey, 2001, papers from the Conference on Webs held in Toulouse, December 1996, Edited by J. Grifone and E. Salem.

[11] P. T. Nagy, Invariant tensorfields and the canonical connection of a 3-web, Aequationes Mathematicae 35 (1988), no. 1, 31-44.

[12] A. Nijenhuis, Jacobi-type identities for bilinear differential concomitants of certain tensor fields. I, II, Indagationes Mathematicae 17 (1955), 390-403.

[13] A. Panasyuk, Veronese webs for bi-Hamiltonian structures of higher corank, Poisson Geometry (Warsaw, 1998), Banach Center Publications, vol. 51, Polish Acad. Sci., Warsaw, 2000, pp. 251261.

[14] _ On integrability of generalized Veronese curves of distributions, Reports on Mathematical Physics 50 (2002), no. 3, 291-297.

[15] F.-J. Turiel, Classification locale simultanée de deux formes symplectiques compatibles, Manuscripta Mathematica 82 (1994), no. 3-4, 349-362.

[16] _ $C^{\infty}$-classification des germes de tissus de Veronese, Comptes Rendus de l'Académie des Sciences. Series I. Mathematics 329 (1999), no. 5, 425-428.

[17] — $C^{\infty}$-équivalence entre tissus de Veronese et structures bihamiltoniennes, Comptes Rendus de l'Académie des Sciences. Series I. Mathematics 328 (1999), no. 10, 891-894.

[18] _ Tissus de Veronese analytiques de codimension supérieure et structures bihamiltoniennes, Comptes Rendus de l'Académie des Sciences. Series I. Mathematics 331 (2000), no. 1, 61-64.

T. B. Bouetou: Département de Mathématique et Génie Informatique, Ecole Nationale Supérieure Polytechnique, Université de Yaoundé I, BP 8390, Yaoundé, Cameroon

E-mail address: tbouetou@darboux.math.univ-montp2.fr

J. P. Dufour: Département de Mathématiques, Université Montpellier 2, Case Courrier 051, Place Eugène Bataillon, 34095 Montpellier Cedex 5, France

E-mail address: dufourj@math.univ-montp2.fr 


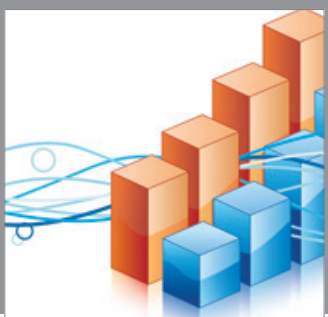

Advances in

Operations Research

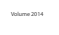

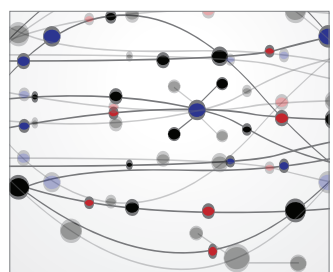

\section{The Scientific} World Journal
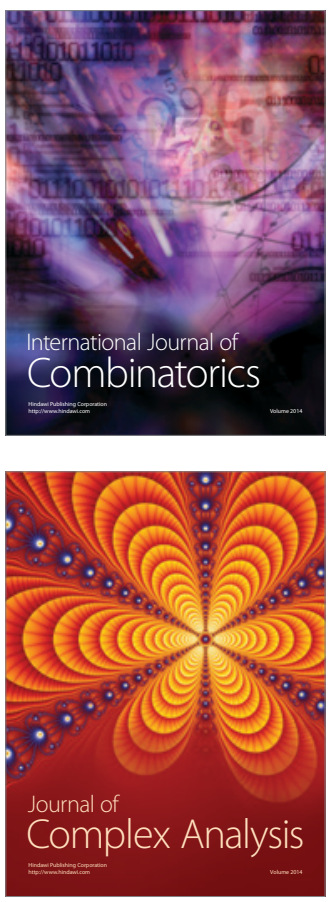

International Journal of

Mathematics and

Mathematical

Sciences
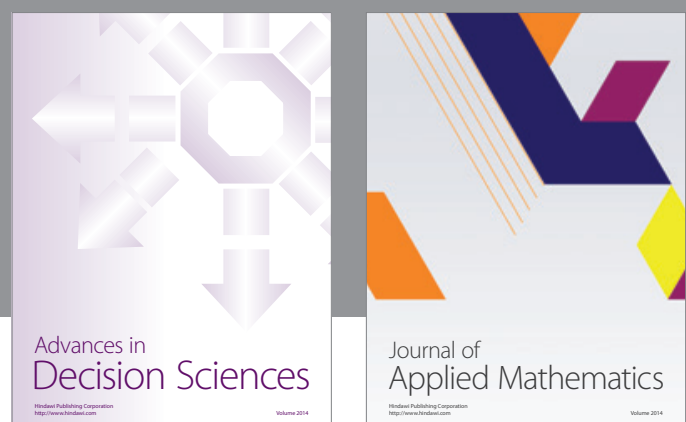

Journal of

Applied Mathematics
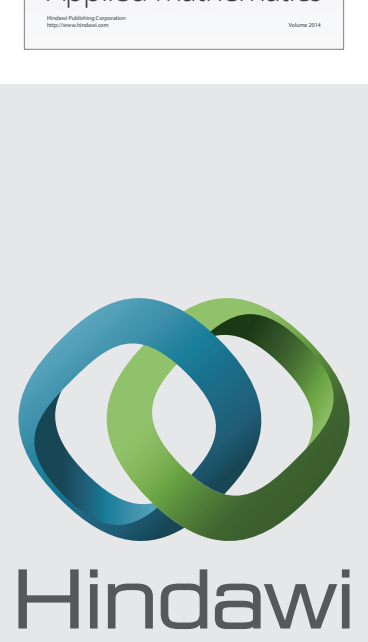

Submit your manuscripts at http://www.hindawi.com
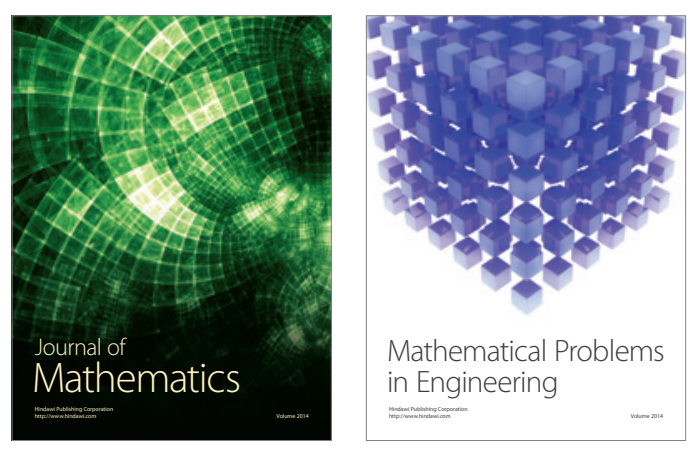

Mathematical Problems in Engineering
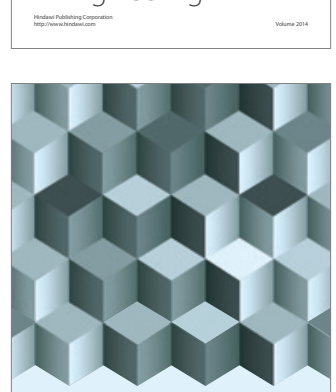

Journal of

Function Spaces
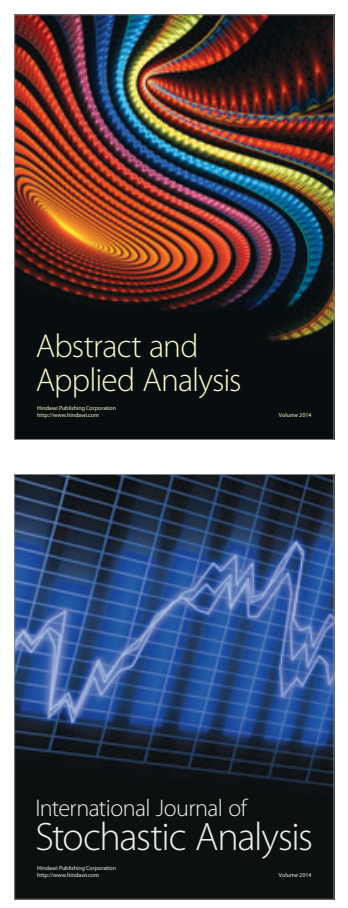

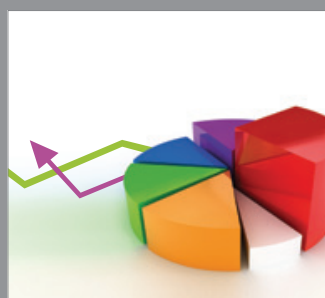

ournal of

Probability and Statistics

Promensencen
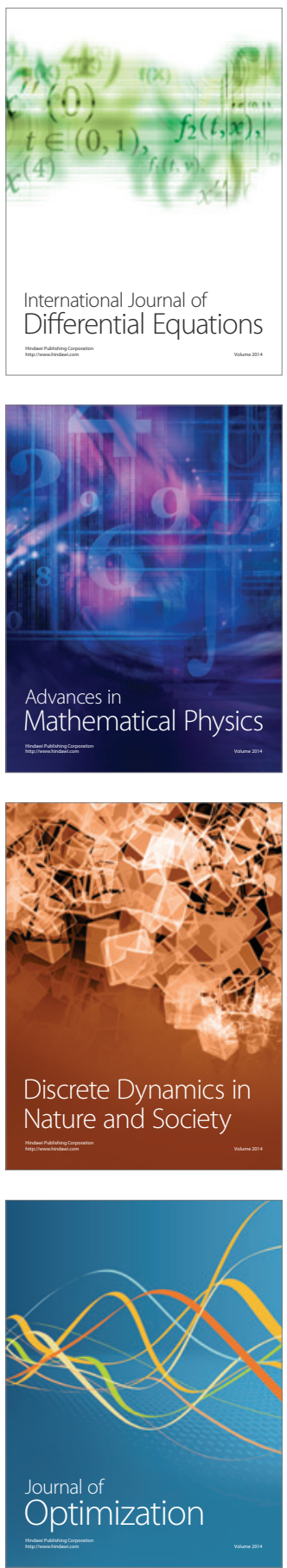